\title{
Impressions of Osteopathic Neurosurgeon on Preparedness for Practice: Survey Results from the American College of Osteopathic Surgeons
}

James Brazdzionis ${ }^{1}$, Harjyot Toor ${ }^{1}$, Tye Patchana ${ }^{1}$, James G. Wiginton IV ${ }^{1}$, Raed Sweiss ${ }^{1}$, Margaret Rose Wacker $^{2}$, Vladimir Cortez ${ }^{3}$, Dan E. Miulli ${ }^{1}$

1. Neurosurgery, Riverside University Health System Medical Center, Moreno Valley, USA 2. Neurosurgery, Arrowhead Regional Medical Center, Colton, USA 3. Neurosurgery, Desert Regional Medical Center, Palm Springs, USA

Corresponding author: James G. Wiginton IV, chipwiginton@me.com

\section{Abstract}

\section{Introduction}

Neurosurgeons trained in the US are rigorously educated on the surgical management of neurosurgical conditions. These neurosurgeons have been trained through one of two avenues: the Accreditation Council for Graduate Medical Education (ACGME) or the American Osteopathic Association (AOA). With the formation of a single accreditation system from the AOA and ACGME accrediting bodies and significant changes introduced in the training of neurosurgeons from both bodies, we sought to identify common practice parameters and perceptions of preparedness of AOA-trained neurosurgeons.

\section{Methods}

A survey was conducted through the neurosurgery section of the American College of Osteopathic Surgeons (ACOS), requesting responses from attending neurosurgeons who completed AOA neurosurgery residency. Responses were obtained through an anonymous, web-based system using single-select multiple-choice questions.

\section{Results}

In total, 52 neurosurgeons participated in the survey. The majority of the 52 respondents practiced in nonacademic settings in urban areas and were exposed to a wide variety of practice environments in terms of case volume and clinical responsibilities. Significantly, $96.15 \%$ of the respondents said they felt adequately prepared for neurosurgical practice after their AOA training.

\section{Conclusion}

Overall, this study highlights both the similarities and variances in practices of osteopathic neurosurgeons. The majority of the participants feel that their training has appropriately prepared them for practice and they are skilled surgeons capable of caring for the safety and well-being of numerous patients in a variety of settings. Most of them practice primarily in private-practice settings at urban centers. Overall, osteopathic neurosurgeons trained in AOA programs report that their training has equipped them well for careers in neurosurgery.

Keywords: neurosurgery, medical education, liability, single accreditation, osteopathic, training, perception, survey, preparedness, aoa

\section{Introduction}

Neurosurgical training in the US is a well-developed and structured academic milieu to train surgeons at providing optimal care for surgical neurological conditions. Historically, in the United States, there have been two avenues for training and board certification for neurosurgeons: neurosurgical residencies at the Accreditation Council for Graduate Medical Education (ACGME) and the American Osteopathic Association (AOA). In light of the planned formation of a single accreditation from the AOA and ACGME residencies and the increasingly intertwined practices of the osteopathic- and allopathic-trained neurosurgeons, we wished to conduct a survey to identify common factors in practice as well as perceptions of preparedness for AOAtrained neurosurgeons. Single accreditation will likely bring changes to the way osteopathic neurosurgery residencies have traditionally operated [1]. Neurosurgeons have been practicing for many years after training through both the accrediting bodies, and support for investigations of preparedness for neurosurgeons have been promoted by multiple neurosurgical organizations. However, there is a dearth of significant data about these aspects. The only identified study investigating preparedness among ACGMEtrained neurosurgeons is by Mazzola et al. [2]. We believe ours is the first paper to investigate preparedness to practice through osteopathic training in neurosurgeons. 


\section{Cureus}

\section{Materials And Methods}

Members of the neurosurgery division of American College of Osteopathic Surgeons (ACOS) who had completed osteopathic neurosurgical training in an AOA-accredited neurosurgical residency were invited to complete an anonymous survey. Invitations for the survey were sent out via email to the aforementioned neurosurgeons on behalf of ACOS. One reminder email was sent out as well to each of the recipients to encourage participation. Additionally, the survey was advertised on the ACOS website. The survey was completed through an anonymous, online platform and remained open for two months. The survey questions were multiple choice with single-selection answers. Physicians were able to defer answering questions if they so chose.

\section{Results}

Of the 195 Osteopathic neurosurgeons who are ACOS members, 52 responded to the anonymous questionnaire. All of them responded to all of the questions with the exception of questions 5, 6, 11, and 13. Of these skipped questions, there was one skipped response for questions

5,6 , and 11 and two skipped responses for question 13. Responses to questions are displayed in a graphical format below (Figures 1-4) and in table format in the Appendix (Table 1).

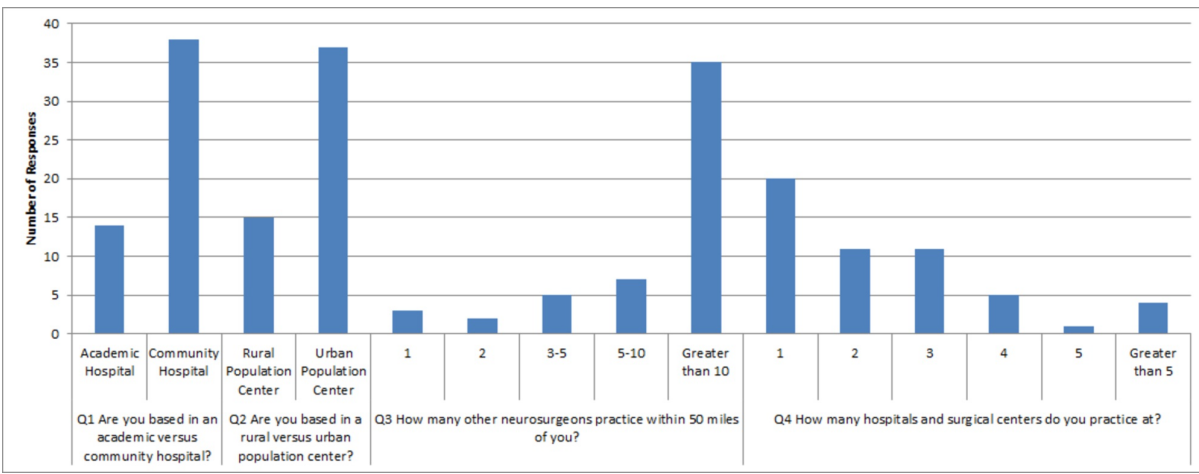

FIGURE 1: Graphical representation of survey responses for questions one through four

Q: question

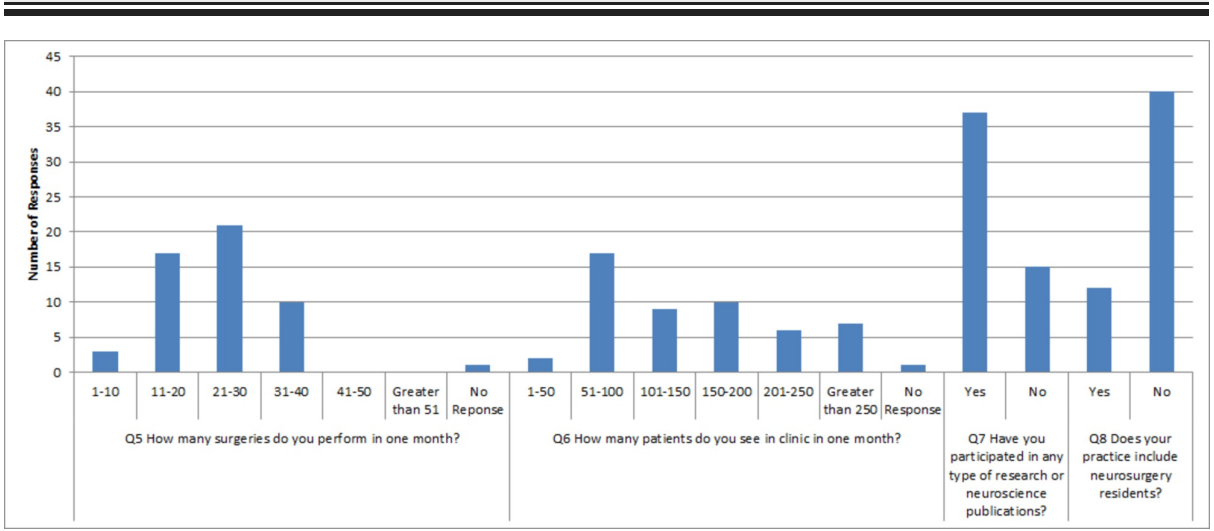

FIGURE 2: Graphical representation of survey responses for questions five through eight

Q: question 


\section{Cureus}

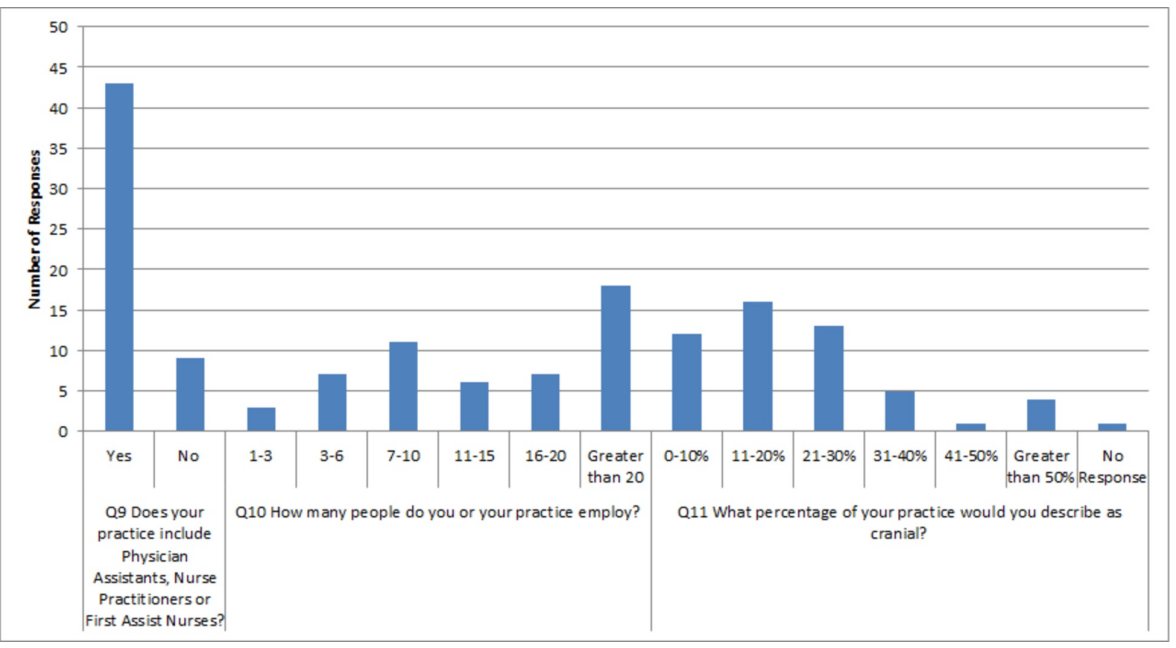

FIGURE 3: Graphical representation of survey responses for questions nine through eleven

Q: question

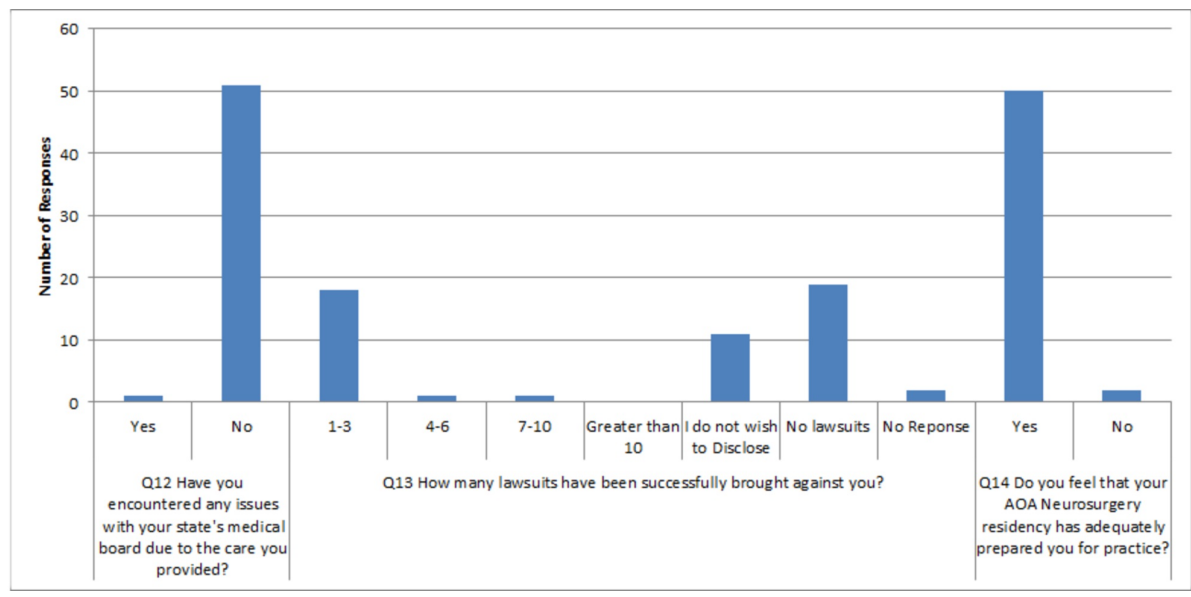

FIGURE 4: Graphical representation of survey responses for questions twelve through fourteen

Q: question

\section{Discussion}

This survey evaluated impressions of neurosurgical training from AOA-board certified neurosurgeons trained in AOA residencies in the US. Overall, these individuals tend to practice in community settings in urban population centers (Figure 1). The majority practice at more than one surgical center or hospital. The most common response of these neurosurgeons indicates that they usually perform anywhere from 21 to 30 surgeries per month, but occasionally tend to range anywhere from 11 to 40 surgeries per month. Clinically, they see a variable number of patients in clinics, with the most common response stating anywhere from 51 to 100 per month. Many are academically oriented with $71.15 \%$ participating in research; however, they do not tend to have neurosurgical-residency involvement. The majority ( $82.69 \%)$ employ mid-level providers or first-assist nurses. Practice sizes are varied and diverse. However, $80.39 \%$ perform $30 \%$ or less cranial neurosurgery in their practice (Table 1).

AOA-trained neurosurgeons surveyed are safe practitioners with only one responder having encountered any issue with their state licensing board due to issue with the care provided (Table 1). The most frequent response in regards to lawsuits was "no successful lawsuits being levied against the neurosurgeon" (38\%). However, 11 neurosurgeons (22\%) specifically stated that they did not wish to disclose anything regarding this, and another two responders skipped this question altogether. This is important to note as a recent study demonstrated that approximately $19.1 \%$ of neurosurgeons face lawsuits each year $[3,4]$. Overall, 


\section{Cureus}

Q5. How many surgeries do you perform in one month?

1-10

3

$5.88 \%$

$11-20$

17

$33.33 \%$

21-30

21

$41.18 \%$

$31-40$

10

$19.61 \%$

$41-50$

$0.00 \%$

Greater than 51

0

$0.00 \%$

No Response

1

Q6. How many patients do you see in clinic in one month?

1-50

2

$3.92 \%$

51-100

17

$33.33 \%$

101-150

9

$17.65 \%$

$150-200$

10

$19.61 \%$

201-250

6

$11.76 \%$

Greater than 250

7

$13.73 \%$

No Response

1

Q7. Have you participated in any type of research or neuroscience publications?

Yes

No

37

15

Q8. Does your practice include neurosurgery residents?

Yes

$28.85 \%$

Q9. Does your practice include physician assistants, nurse practitioners, or first-assist nurses?

Yes

43

No

Q10. How many people do you or your practice employ?

$1-3$

3-6

$7-10$

11-15

$16-20$

Greater than 20

Q11. What percentage of your practice would you describe as cranial?

$0-10 \%$

$11-20 \%$

$21-30 \%$

$31-40 \%$

$41-50 \%$
12

16

5

3

7

11

6

7

18

1
$82.69 \%$

$17.31 \%$

$5.77 \%$

$13.46 \%$

$21.15 \%$

$11.54 \%$

$13.46 \%$

$34.62 \%$

$23.53 \%$

$31.37 \%$

$25.49 \%$

$9.80 \%$

$1.96 \%$ 


\section{Cureus}

Greater than $50 \%$

4

No Response

Q12. Have you encountered any issues with your state's medical board due to the care you provided?

Yes

No

Q13. How many lawsuits have been successfully brought against you?

$1-3$

4-6

$7-10$

Greater than 10

I do not wish to Disclose

No lawsuits

No Response
18

1

1

0

11

19

2
1

51

$1.92 \%$

$98.08 \%$

Q14. Do you feel that your AOA Neurosurgery residency has adequately prepared you for practice?

Yes

No
50

2
$96.15 \%$

$3.85 \%$

TABLE 1: Tabulation for responses from surveyed osteopathic neurosurgeons. Calculated percentage of responses do not include those of people who did not respond to the question

Q: question; AOA: American Osteopathic Association

\section{Additional Information}

\section{Disclosures}

Human subjects: All authors have confirmed that this study did not involve human participants or tissue. Animal subjects: All authors have confirmed that this study did not involve animal subjects or tissue. Conflicts of interest: In compliance with the ICMJE uniform disclosure form, all authors declare the following: Payment/services info: All authors have declared that no financial support was received from any organization for the submitted work. Financial relationships: All authors have declared that they have no financial relationships at present or within the previous three years with any organizations that might have an interest in the submitted work. Other relationships: All authors have declared that there are no other relationships or activities that could appear to have influenced the submitted work.

\section{References}

1. Miller T, Jarvis J, Mitchell K, Miser WF: The single accreditation system: more than a merger . Ann Fam Med. 2016, 14:383-4. 10.1370/afm.1958

2. Mazzola CA, Lobel DA, Krishnamurthy S, Bloomgarden GM, Benzil DL: Efficacy of neurosurgery resident education in the new millennium: the 2008 council of state neurosurgical societies post-residency survey results. Neurosurgery. 2010, 67:225-32. 10.1227/01.NEU.0000372206.41812.23

3. Jena AB, Seabury S, Lakdawalla D, Chandra A: Malpractice risk according to physician specialty . N Engl J Med. 2011, 365:629-36. 10.1056/NEJMsa1012370

4. Thomas R, Gupta R, Griessenauer CJ, et al.: Medical malpractice in neurosurgery: a comprehensive analysis . World Neurosurg. 2018, 110:e552-559. 10.1016/j.wneu.2017.11.051

5. Cohen-Gadol AA, Piepgras DG, Krishnamurthy S, Fessler RD: Resident duty hours reform: results of a national survey of the program directors and residents in neurosurgery training programs. Neurosurgery. 2005, 56:398-403. 10.1227/01.NEU.0000147999.64356.57 